\title{
Balanced incomplete blocks to control individual differences
}

\author{
HERBERT D. KIMMEL, JUDITH A. BECKER, and MICHAEL J. BEVILL \\ University of South Florida, Tampa, Florida
}

\begin{abstract}
Balanced incomplete block designs are used when there are reasons that preclude the use of repeated measurements designs, particularly when there are at least two factors, each with several levels. However, these designs are usually not used when interaction effects are of interest, because they involve the confounding of the interactions with subject or group differences. This paper describes special computational procedures that can be employed to obtain partial, but unconfounded, information about interaction effects.
\end{abstract}

The repeated measurements design is probably the most common procedure employed in psychological research to control individual differences experimentally. This design permits subjects to serve as their own controls and to receive all of the experimental treatments (or treatment combinations) generated by the independent variables. Assuming that the subjects represent a random sample from some population of interest (e.g., normal human adults), the mixed mathematical model underlying the analysis of variance permits appropriate treatments $X$ subjects interactions to be used to estimate error variance in testing the significance of the various treatment effects.

Three considerations most commonly limit the use of repeated measurements designs. The first of these, the effect of sequence, can usually be dealt with satisfactorily either by counterbalancing all possible sequences across subjects (when the number of treatments is small enough) or by running a reduced set of sequences in a Latin square arrangement, in which each treatment appears only once in each ordinal position in the sequence. Repetitions of such Latin squares, or several different squares, can be employed to achieve the desired overall sample size, which must be a multiple of the order of the Latin square.

The second constraint against repeated measurements designs occurs when the number of treatments is too great to be administered to any single subject because this would be too time-consuming (causing fatigue or reduced motivation), too psychologically complicated, or otherwise impractical. For example, even a relatively simple design that involves only two orthogonal independent variables, each at three levels, generates nine treatment combinations. Depending upon the nature of the behavioral situation, or the task, it may be quite infeasible to administer all nine conditions to a single subject.

A third limitation on the use of a repeated measurements design is that it may reveal to the subjects the purpose of the study. That is, if subjects are given all combina-

Address correspondence to Herbert D. Kimmel, Department of Psychology, University of South Florida, Tampa, FL 33620. tions of the variables of interest, the comparison being made may be obvious and thus bias subjects' responses.

In such cases, it is sometimes possible to administer some, but not all, of the treatments to individual subjects, so that within-subject control of individual differences may still be achieved in the tests of experimental hypotheses. Different subjects or groups of subjects receive different subsets of treatments, but individual differences are still kept out of the error terms needed to test treatment effects. Since such incomplete blocks designs (Cochran \& Cox, 1957) inevitably involve the confounding of some main effects or interactions with subject or group differences, balanced experimental plans and special computational techniques must be employed to obtain unconfounded estimates of the necessary mean squares. In this paper, computational procedures are outlined for obtaining partial, but unconfounded, information regarding the row $\times$ column interaction in a $3 \times 3$ factorial experiment run in balanced incomplete blocks. Main effects are unconfounded, but the row $\times$ column interaction is totally confounded with group differences and can only be obtained using special techniques. This design has been described previously, to illustrate deliberate confounding of the interaction in a $3 \times 3$ factorial (Cochran \& Cox, 1957), but the computational technique for computing the interaction sum of squares is novel. Indeed, Cochran and Cox suggested that the design should not be used if information regarding the interaction is required.

Both Winer (1971) and Kirk (1982) also presented plans for designs of this type, but both used abstruse modular arithmetic methods for identifying the sets of treatment combinations to be administered to each individual subject, rather than the present, more broadly comprehensible, technique. Neither Winer nor Kirk presented an actual experimental example, as is done here, and their modular arithmetic methods for group identification do not make it clear that there are two sets of groupings that are orthogonal to each other and to the row and column effects.

This design was recently employed in an experiment by Becker, Bevill, and Kimmel (1988) on the effects of 
request form and speaker status on the interpretation of requests. College-aged subjects read scenarios, in each of which a speaker made a request of the subject. The speakers varied in their status or power relative to the subject (i.e., higher, same, lower), and requests varied according to the degree to which the content of the request was made explicit (i.e., quite explicit, explicit, ambiguous), which was a function both of syntactic structure and semantic phrases. The subjects were asked to rate each request on three 5-point Likert-type scales: powerfulweak, rude-polite, and humble-arrogant. Previous research (Becker, 1982, 1986; Ervin-Tripp, 1976) demonstrated that speakers tend to address more explicit requests to lower status listeners and more ambiguous requests to higher status listeners, and that listeners tend to judge explicit requests as rude and judge more ambiguous requests as polite. Becker et al. were interested in the interactive effects of speaker status and request form on listeners' judgments of requests, particularly when the request forms were not those typically used by speakers of particular statuses. They hypothesized that judgments would be a function of both the form of the request and the context in which it was used (i.e., speaker's relative status).

The complexity of the subjects' task and the possibility that the manipulation would be made transparent precluded administering all nine treatment combinations to any one subject. For these reasons, a balanced incomplete blocks design was used, with only three treatments per subject.

Table 1 represents the $3 \times 3$ experimental plan used in this study, with request form in rows and speaker status in columns. The levels of each factor are identified in the table as 0,1 , or 2 (indicating increasing explicitness of the request and increasing status of speaker), for consistency with Cochran and Cox's (1957) presentation of this design. A Greco-Latin square has been superimposed on the $3 \times 3$ factorial table, for reasons given below.

A between-groups effect with $2 d f$ that is orthogonal to both the row and column effects is defined by the following comparison of the three sets of cell means associated with the different Latin letters:

$$
\begin{aligned}
& A=(00)+(12)=(21) \\
& B=(01)+(10)=(22) \\
& C=(02)+(11)+(20)
\end{aligned}
$$

Thus the Latin letter effect necessarily comprises 2 of the $4 d f$ for the row $\times$ column interaction (since it is a

Table 1

$3 \times 3$ Experimental Plan with Superimposed Greco-Latin Squares

\begin{tabular}{cccc}
\hline & \multicolumn{3}{c}{ Speaker Status } \\
\cline { 2 - 4 } Request Explicitness & 0 & 1 & 2 \\
\hline 0 & $00(\mathrm{~A}, \alpha)$ & $01(\mathrm{~B}, \beta)$ & $02(\mathrm{C}, \gamma)$ \\
1 & $10(\mathrm{~B}, \gamma)$ & $11(\mathrm{C} \alpha)$ & $12(\mathrm{~A}, \beta)$ \\
2 & $20(\mathrm{C}, \beta)$ & $21(\mathrm{~A}, \gamma)$ & $22(\mathrm{~B}, \alpha)$ \\
\hline
\end{tabular}

Note-The order $0,1,2$ indicates increasing explicitness of the request and increasing status of the speaker. between-groups effect that is neither the row nor the column effect).

The other between-groups effect with $2 d f$ that is orthogonal to the row and column effects (again by the Latin square principle) and to the Latin letter effect is defined by the following comparison of the three sets of cell means associated with the different Greek letters.

$$
\begin{aligned}
& \alpha=(00)+(11)+(22) \\
& \beta=(01)+(12)+(20) \\
& \gamma=(02)+(10)+(21)
\end{aligned}
$$

The Greek letter effect thus comprises the remaining 2 of the $4 d f$ for the row $\times$ column interaction.

The subjects were assigned randomly to the six different groups identified by the three Latin letter groupings of treatments and the three Greek letter groupings of treatments. An individual subject in one of these six groups received only those three treatment combinations associated with his/her Latin or Greek letter groupings. The $5 d f$ associated with differences among these six groups consist of $4 d f$ for the row $\times$ column interaction plus the $1-d f$ difference between the Latin and Greek halves of the experiment (sometimes called square uniqueness). Order of administration of the three treatments received by each subject may be balanced across subjects within each of the six groups. (With multiples of $n=6$ in each group, all of the six possible sequences of three treatments were used equally often.)

As noted above, the overall row $\times$ column interaction is completely confounded with differences among the different groups of subjects. However, because the experimental plan is balanced, even though 2 of the $4 d f$ for the row $x$ column interaction in the Latin letter half of the experiment are confounded with the Latin letter grouping, the other $2 d f$ for the interaction in this half of the experiment are unconfounded, because the Greek letter grouping involved different subjects. The reverse is true in the Greek letter half of the experiment. Here, 2 of the $4 d f$ for the row $\times$ column interaction are confounded with the Greek letter grouping, but the other $2 d f$ for the interaction in this half of the experiment are unconfounded, because the Latin letter grouping involved different subjects. Thus, half of the information regarding the interaction may be recovered separately from within the two halves of the experiment.

Within the Latin letter half of the experiment, the row $x$ column interaction is computed in an orthodox fashion. From this 4-df sum of squares, the 2-df sum of squares for the Latin letter factor must be subtracted. The remaining $2 d f$ for the interaction are unconfounded. Within the Greek letter half of the experiment, the row $\times$ column interaction is computed similarly. From this $4-d f$ sum of squares, the 2-df sum of squares for the Greek letter factor must be subtracted. The remaining $2 d f$ for the interaction are unconfounded. Note that data from only half of the subjects are employed in computing the necessary terms to obtain each of these two separate unconfounded 
interaction sums of squares. In the first case, only data from the three groups of subjects who received the Latin letter sets of treatments are used, and in the second case only data from the three groups of subjects who received the Greek letter sets of treatments are used. The two separate sums of squares may be pooled for testing the significance of the row $\times$ column interaction, using the standard error term from the overall analysis of variance, the row $\times$ columns $\times$ subjects mean square, unless the two separate estimates of the interaction are significantly heterogeneous. In this case, each may be tested separately with the appropriate error term computed within its own half of the experiment. Under ordinary circumstances, there is no reason why the two separate estimates of the row $\times$ column interaction should be heterogeneous.

In any case, it is clear that an unconfounded evaluation of the row $\times$ column interaction, based on half the information, may be made when balanced incomplete blocks have been used to control individual differences, even though the overall interaction is completely confounded with group differences in this design. Depending upon the importance of the row $\times$ column interaction, $50 \%$ loss of information may be judged to be too severe, in which case an unconfounded design would have to be used. On the other hand, if subject differences constitute a major source of error, as was believed to be the case in the present example, a balanced incomplete blocks design offers a reasonable compromise.

\section{REFERENCES}

BECKER, J. A. (1982). Children's strategic use of requests to mark and manipulate social status. In S. Kuczaj (Ed.), Language development: Language, thought, and culture (p. 1-35). Hillsdale, NJ: Erlbaum. BECKER, J. A. (1986). Bossy and nice requests: Children's production and interpretation. Merrill-Palmer Quarterly, 32, 393-413.

Becker, J. A., BeviLl, M. J., \& Kimmel, H. D. (1988). [The interactive effects of request form and speaker status on judgments of requests]. Unpublished raw data.

Cochran, W., Cox, G. (1957). Experimental designs (2nd ed.). New York: Wiley.

ERvin-TrIPP, S. (1976). Is Sybil there?: The structure of some American English directives. Language in Society, 5, 25-66.

KIRK, R. E. (1982). Experimental designs: Procedures for the behavioral sciences. Belmont, CA: Brooks/Cole.

WINER, B. J. (1971). Statistical principles in experimental design. New York: McGraw-Hill.

(Manuscript received July 7, 1987;

revision accepted for publication December 22, 1987.) 